\title{
A model of human response to workload stress
}

\author{
MOIRA LEMAY, FRANCES LAYTON, and DAVID J. TOWNSEND \\ Montclair State College, Upper Montclair, New Jersey
}

\begin{abstract}
When faced with decreased time to complete a task, operators often hold performance constant. When workload is excessive, this is not possible and operators may speed work and ignore errors or fail to finish the task. In this experiment, we forced a choice among policies by decreasing time allowed for a task until it could not be completed. Operators established a baseline time to perform a standard task, and times were decreased to $80 \%, 60 \%, 50 \%, 40 \%$, and $30 \%$ of this baseline. Operators were able to decrease time per subtask by $20 \%$, but no further decreases were observed as time demands increased. Errors remained constant over all conditions. The data support the view that increases in task demand produce increases in resources that are adequate for small demand increases but insufficient for great task demands.
\end{abstract}

Modern automated systems often require that a variety of tasks be assigned to a human operator, since automation of routine tasks itself creates a need for supervisory functions (Moran, 1983). Since each operator controls a large segment of an automated system's operations, the impact of operator performance is greatly increased. The time an operator takes to perform a task, and the possibility of operator errors, can become important, and possibly limiting, factors in computer systems. The study of interactive computer systems has received considerable attention in the literature (Kantowitz \& Sorkin, 1983; Schneiderman, 1980). In particular, the task of text editing has been analyzed as a representative interactive computer task (Card, Moran, \& Newell, 1983; Ledgard, Whiteside, Seymour, \& Singer, 1980). Card et al. (1983) have proposed a model of operator behavior in computer tasks in an attempt to predict an individual's actual sequence of operations, along with the time it will take that individual to do the job.

A previous study on workload measurement in a large interactive computer system (LeMay \& Hird, 1986) has indicated that workload can be assessed on the subtask level, thus allowing for separate assessment of subtask and whole task times. This makes it possible to increase objective load on an operator's processing capacity by reducing the time that is allowed for a whole task. Increased operator output can then still be measured by the time required per subtask, even though total task time is determined by the experimental situation. This yields a time line analysis measure of workload (Wickens, 1986).

Workload can also be measured by obtaining subjective ratings of mental load, introducing a secondary task to gauge its effect on the primary task, and measuring certain physiological responses. Introducing a secondary task has recently taken on more importance, because au-

\footnotetext{
The authors wish to thank Carl Bredlau for the preparation of the keystroke timing program used in this experiment. Requests for reprints should be addressed to Moira LeMay, Department of Psychology, Montclair State College, Upper Montclair, NJ 07043.
}

tomation imposes demands from multiple sources on a single operator's capacity. Thus, models of operator processing capacity such as the undifferentiated capacity models of Kahneman (1973) and Moray (1979) or the multiple resource model of Wickens $(1984,1986)$ have been concerned with changes in performance among time-shared tasks. However, the sequential nature of many computer tasks lends them well to a time line subtask analysis, in which a representative task is divided into subtasks, and operator workload is measured as the ratio of time required to time allowed for a subtask.

Of course, a major advantage of computer systems is the ease of error correction, and an advantage of human operators is that they detect and correct their own errors. Thus, the combination of the human operator and the computer results in few overall significant errors under normal circumstances. However, the introduction of an increased workload did result in increased errors in LeMay \& Hird (1986), and thereby increased the risk of a significant error's going undetected. Thus, workload measures and workload effects are not trivial.

This is not true, of course, in word processing systems, where undetected errors may result merely in inconvenience. However, word processing has been considered a prototypical interactive computer task (Card, Moran, \& Newell, 1983; Ledgard, Whiteside, Seymour, \& Singer, 1980 ), and principles developed on the basis of this task can probably be generalized to other computer tasks if the unique properties of the task are taken into consideration. In the present study, we examined the relationship between increases in workload and the policies an operator employs in performing a standard task. When faced with an increase in workload, an operator has three choices:

1. Increase working speed to increase output, and ignore increased uncorrected errors.

2 . Work as carefully as usual and ignore the possibility of not getting done on time (a "haste makes waste" policy).

3. Use some combination of these, involving a speedup of work along with some attention to error correction. 
The best policy is obviously a function of the task and its priorities. Nevertheless, it should prove useful to determine which policy leads to the greatest productivity. In previous studies of workload (Moray, 1979; O'Donnell \& Eggemeier, 1986; Wierwille, Williges, \& Schiflet, 1979), it has been found that, under increased workload, operators tend to adapt to the task and hold performance constant, indicating that the third policy may be followed most often. In the present study, we attempted to force a choice of policy by decreasing the time allowed for a task until it could not be completed. Thus, the policy pursued by an operator should become evident in (1) decreased time required for each subtask and increased errors (Policy 1 above), (2) increased time per subtask and no increase in errors (Policy 2), or (3) decreased time for each subtask, with little or no increase in errors (Policy 3). It was hypothesized that operators might increase their output of work while experiencing an increase in workload up to a certain decreased level of allowed time without any increase in errors or changes in policy; that is, they would adopt Policy 3. Beyond that point, operator errors would start to increase and time required for each subtask would level off, causing the workload ratio to increase at a slower rate.

\section{METHOD}

\section{Subjects}

Ten experienced computer users, drawn from a college student and faculty population, served as subjects in the experiment. All were familiar with the use of WordPerfect.

\section{Apparatus}

The experiment was run on an IBM XT, programmed with WordPerfect 4.2. An additional program recorded and timed each keystroke.
Seven 46-line segments of text with an error density of .5 modifications per line, marked in red ink, were prepared and printed out as experimental materials. Errors, which were inserted randomly in the texts, consisted of "type overs" of no more than two letters or punctuation marks. These constituted the subtasks of each total computer task. The completion of a total task meant fulfilling the goal of producing a text segment on the screen with all the marked errors corrected.

\section{Procedure}

The computer was loaded with WordPerfect and the keystroke timing program, as well as a text segment with uncorrected errors. A printout of that segment was face down beside the keyboard. A clock and an index card containing a 7-point time stress scale, where 1 indicated low time stress and 7 indicated high time stress, were plainly visible to the operator.

The first trial was a practice trial. This was followed by a trial in which the operator performed the task at a "comfortable" speed with no time pressure. At the end of each correction, operators pressed one of the number keys on the keyboard, giving a rating on a scale from 1 to 7 for the amount of time stress involved in that correction (subtask); this was used as a timing device, as described by LeMay and Hird (1986). At the end of the trial, the operators were informed of the amount of time they had taken for the whole task, and this established a baseline of $100 \%$ for that operator, from which time decrements of $80 \%$, $60 \%, 50 \%, 40 \%$, and $30 \%$ were calculated.

\section{RESULTS}

The mean of the subjective time stress ratings $(R)$ was obtained for each time decrement, as is shown in Figure 1. Subjective ratings of time stress increased as time allowed decreased $[F(5,40)=5.40, p<.01]$. The increase in subjective ratings is not as great as might have been expected for such severe reductions in the time allowed for the task.

The number of subtasks completed in the $100 \%$ trial was divided into the amount of time required to complete it to obtain the mean time required per subtask in that trial.

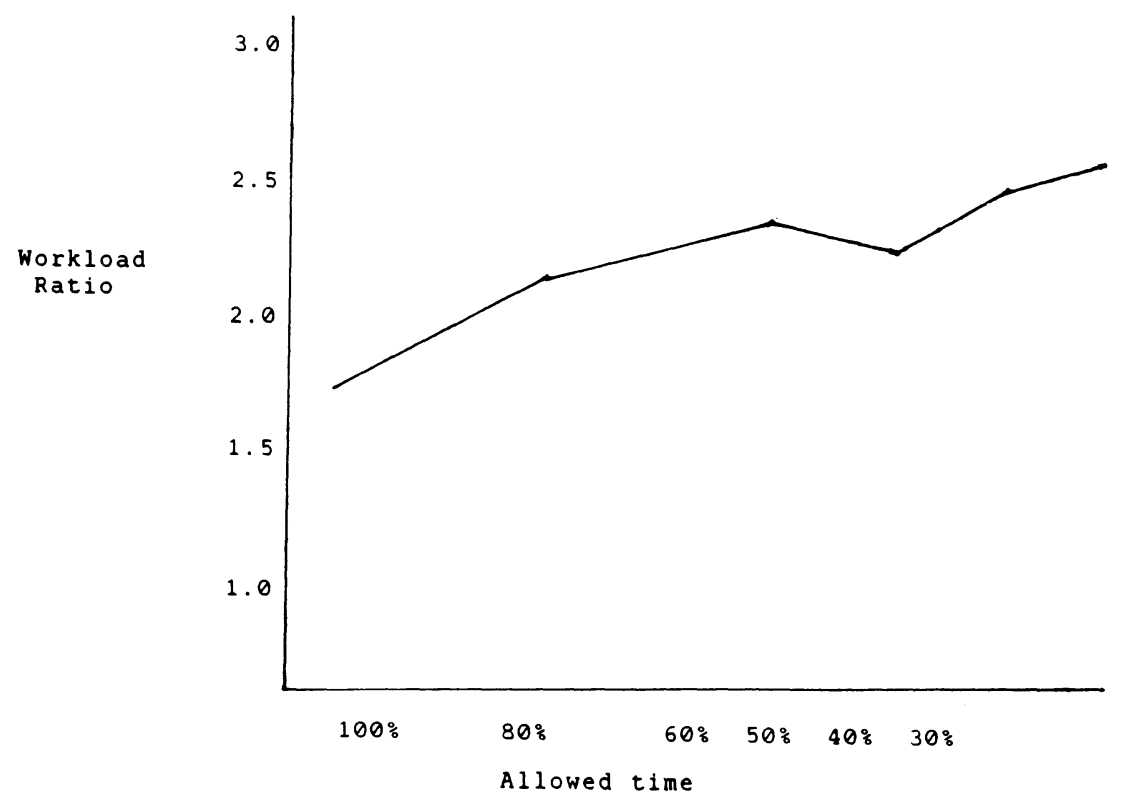

Figure 1. Mean subjective ratings of subtasks in a text editing task as a function of decreases in time allowed (Ta) to do the task. 


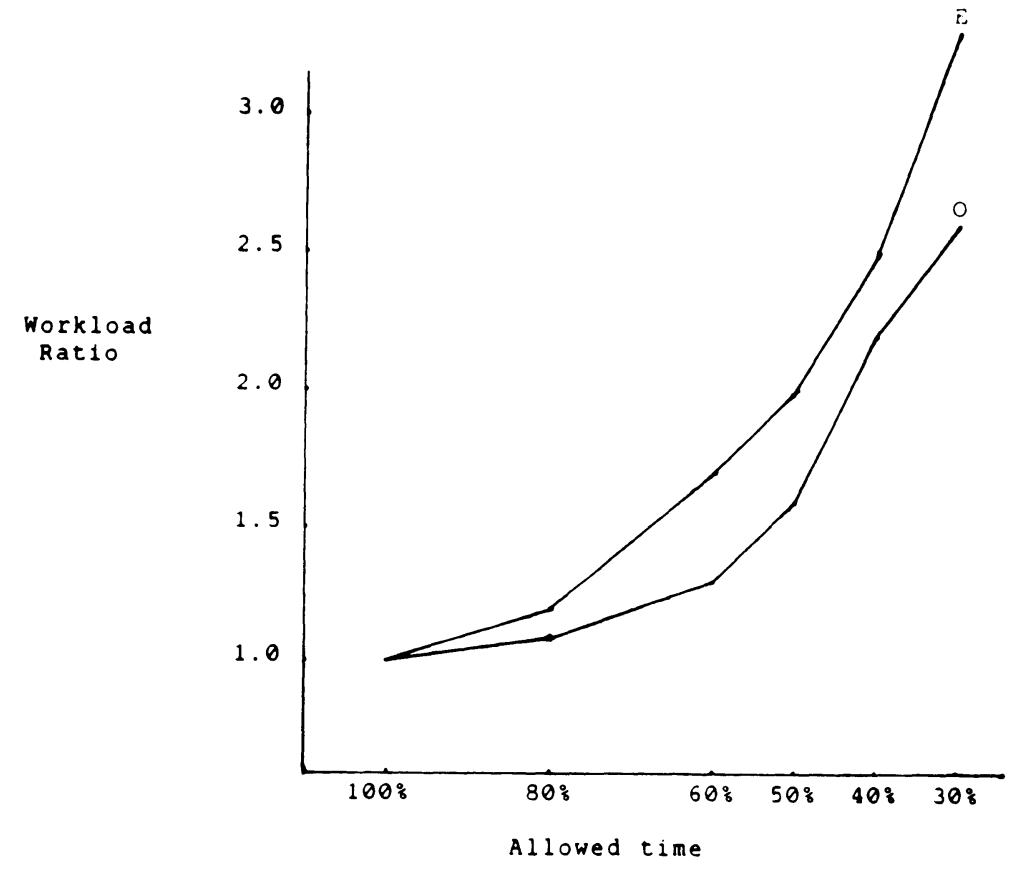

Figure 2. Mean workload (W) as a function of decreasing allowed time for a subtask.

Since no time pressure was put on the users in this trial, this was also the time allowed, and so the workload ratio was one for that trial. This time was then used as the basis for determining the (shortened) time allowed in subsequent trials.

Mean workload ratio was obtained for each shortened allowed time. Allowed time had a significant effect on workload ratio $[F(4,32)=27.88, p<.01]$. This is illustrated in Figure 2, where both an expected and an obtained workload ratio are plotted as a function of allowed time. The expected workload ratio was calculated by dividing the time required for the $100 \%$ trial by the time allowed for each shortened trial. The obtained workload ratio is a more or less constant percentage of the expected ratio. Thus, operators increased their output per unit time by a constant amount, regardless of increasing demand. The subjects decreased required time per unit task to $80 \%$ of normal, but they did not decrease it any further as demands increased.

Decreasing allowed time had no effect on the mean number of uncorrected errors $(F<1)$. A somewhat higher error rate was observed in the first, $100 \%$, trial, but this may be a practice effect. All the error rates obtained were below $1 \%$.

\section{DISCUSSION}

Operator workload, as measured by both subjective ratings and the workload ratio, increases with the imposition of decreasing amounts of time allowed for a short task. This is to be expected. However, it is the nature of the relationship that is of interest. Despite the fact that very large demands were made on operators in this study, there was only a very shallow rise in subjective workload ratings. Evidently, com- petent operators in an interactive computer task do not experience a heavy subjective workload even under severe time pressure.

Operators in the present study did not seem to reach the point of "resonance loading" suggested by Tulga and Sheridan (1980), despite the time pressure. The idea of "resonance loading" suggests that, when workload reaches unity and beyond, there is a point at which subjective stress reaches a maximum and after which it decreases as the operator realizes the impossibility of task completion. This was certainly the case in the experiment reported here, since workload was well beyond unity. Instead of reaching such a point, subjective ratings continued to increase as further demands were made, in spite of the fact that the policy adopted by the operator was clearly a modified version of Policy 2 mentioned above, since there was no increase in errors. Operators speeded up their performance so that subtasks were performed in about $80 \%$ of the time they would normally take, regardless of how fast they were asked to perform them. Taken together, these findings indicate that operators can increase their output by small amounts when there is a small increase in subjective stress, but that they are probably not able to increase it any further, since the only effect of increasing demand is an increase in subjective workload ratings. If operators are simply unwilling to work as demanded, there should be no significant increase in ratings.

Thus, it seems that operators have a limited ability to compensate for increasing task demands by increasing their output per unit time. This has implications for many typical user tasks, which frequently involve long periods of very low activity level interspersed with short bursts of greatly increased activity. If a task requires a sudden increase in output, by how much can user production be expected to increase, as expressed in terms of a normal output rate, and what is the effect of the increase on error rate? This study seems to indicate that operators can decrease time to perform a task with virtually no error increase, but that this decrease is limited to a percentage of normal task time. Thus, operators have a limited capacity to increase work output in response to increasing task demands.

The tasks required in this study always demanded full operator attention on a single information-processing channel. If attention is thought of as a single resource (Kahneman, 1973; Moray, 1979), the data support the view that an increase in task demand produces an increase in resources that is adequate for small demand increases but that quickly becomes insufficient for greater ones. For operators in this situation, 
increase in output was small indeed, but this may simply be because demands on a voluntary subject in an experiment do not have the same force as real job demands. The point at which operators hold their performance constant might also be expected to vary with task and operator skill level. These possibilities require further investigation.

The findings also show that increasing workload on this task, which might be considered visual-verbal in terms of multiple resource theory (Wickens, 1984), reveals the limits on operator capacity. However, multiple resource theory would predict that, for example, a spatial or auditory task might be performed simultaneously with text editing and interfere with it very little. Informal observation of people at work seems to confirm this, and Derrick (1988) has presented data to show that performance decrements on shared dissimilar tasks were smaller than those on shared tasks that were similar in resource demand. Combining dual, dissimilar tasks with increases in workload on a single task might not produce a much greater decrement than that produced in the present study, if multiple resource theory predictions are upheld. This requires further investigation.

Primary task measures of workload such as the one used here are sometimes seen as insensitive, since operators may use spare capacity to deal with increases in workload while maintaining constant task performance (O'Donnell \& Eggemeier, 1986). This would occur only under moderate workload conditions. In this study, by increasing workload beyond normal output levels, we have presented a technique that could be used with a number of primary task measures to render them sensitive to differing task demands.

The procedure can also be adapted to the common situation in which a very low activity level is interspersed with a burst of greatly increased workload. In this situation, it would be useful to know by how much user output might be expected to increase relative to normal output, and what effects, if any, there would be on error rate. The study reported here presents a method for determining the effects of increased time demands on operators of complex, interactive, computer-based systems. The method must be adapted to each particular job, but it should prove useful in a number of situations.

\section{REFERENCES}

Card, S., Moran, T., \& Newell, A. (1983). The psychology of humancomputer interaction. Hillsdale, NJ: Erlbaum.
DERRICK, W. L. (1988). Dimensions of operator workload. Human Factors, 30, 95-110.

Kahneman, D. (1973). Attention and effort. Englewood Cliffs, NJ: Prentice-Hall.

KANTOWITZ, B., \& SORKIN, R. (1983). Human factors: Understanding people-system relationships. New York: Wiley.

Ledgard, H. F., Whiteside, J. A., Seymour, W., \& Singer, A. (1980). An experiment on human engineering of interactive software. IEEE Transactions on Software Engineering, 6, 602-604.

LEMAY, M., \& HIRD, E. (1986). Operator work load: When is enough enough? Communications of the ACM, 29, 638-642.

Moran, T. (1983, August). Applying psychology to human-computer interaction. Paper presented at the American Psychological Association Workshop on Human-Computer Interaction, Anaheim, CA.

Moray, N. (Ed.) (1979). Mental workload: Its theory and measurement. New York: Plenum.

O'DonNELl, R. D., \& EGGEMEIER, F. T. (1986). Workload assessment methodology. In K. Boff, L. Kaufman, \& J. P. Thomas (Eds.). Handbook of perception and human performance (Vol. 2, pp. 42-1-42-49). New York: Wiley.

SCHNEIDERMAN, B. (1980). Software psychology. Cambridge, MA: Winthrop.

Tulga, M. K., \& Sheridan, T. (1980). Dynamic decisions and work load in multitask supervisory control. IEEE Transactions on Systems, Man, and Cybernetics, SMC-10, 217-232.

Wickens, C. D. (1984). Processing resources in attention. In R. Parasuraman \& R. Davies (Eds.), Varieties of attention (pp. 63102). New York: Academic Press.

WiCKENS, C. D. (1986). Engineering psychology and human performance. Columbus, $\mathrm{OH}$ : Merrill.

Wierwille, W. W., Williges, R. C., \& Schiflet, S. G. (1979). Aircrew workload assessment techniques. In B. O. Hartman \& R. E. McKenzie (Eds.), Survey of methods to assess workload (AGARDograph No. 246, pp. 19-53). Springfield, VA: National Technical Information Service. 